\title{
New boundary element analysis of acoustic problems with the fictitious eigenvalue issue
}

\author{
M. Tanaka ${ }^{1}$, Y. Arai $^{1} \&$ T. Matsumoto ${ }^{2}$ \\ ${ }^{1}$ Department of Mechanical Systems Engineering, \\ Shinshu University, Japan \\ ${ }^{2}$ Division of Mechanical Engineering, \\ Graduate School of Nagoya University, Japan
}

\begin{abstract}
This paper is concerned with a new approach for avoiding the fictitious eigenfrequency problem to boundary element analysis of three-dimensional acoustic problems governed by Helmholtz equation. It is well known that in solving without any care the external acoustic problem which includes internal sub-domains by means of the boundary integral equation, the solution is disturbed at fictitious eigenfrequencies corresponding to the internal sub-domains. The present paper proposes a new boundary element analysis to circumvent such the fictitious eigenfrequency problem, which is an alternative boundary integral equation approach to the Burton-Miller one. The present approach is implemented, and its validity and effectiveness are demonstrated through numerical computation of typical examples.
\end{abstract}

\section{Introduction}

Whenever the acoustic problems which include the sub-domains without vibration are solved by means of the usual boundary integral equation without any care, the so-called fictitious eigenvalue issue is encountered. It is well known that the solution of the external acoustic problem is violated near the eigenfrequencies of the inside sub-domains. In practice, if we locate a few source points in the subdomains without vibration and solve the system of equations by the method of least squares, we can circumvent the above eigenvalue issue [1-3]. Nevertheless, in finding the optimal shapes of acoustic fields, for example, it is almost impossible to apply the above practical mehtod, as the current shape is changing and the final, 
converged solution is obtained in an iterative manner. It is true that we cannot find an appropriate number of external points to be added and their appropriate locations for such problems. There is a remedy of this problem, however, which is called the Burton-Miller method. This method employs a linearly combined boundary integral expression of the usual boundary integral equation (OBIE) and the normal derivative boundary integral equation (NDBIE) multiplied with the coupling parameter $[4,5]$.

The present paper proposes an alternative approach to this method to reduce burden of calculating the coefficients in matrixes of the final system to be solved. If we assume to use higher-order boundary elements, the Burton-Miller expression is used at a smaller number of element nodes, and at the other elemet nodes we employ the NDBIE multipied with the same coupling parameter. Through numerical computations it is demonstrated that the present approarch and the Burton-Miller one provide the almost identical results to circumvent always the fictitious eigenvalue issue.

\section{Theory}

It is assumed that the acoustic problems to be investigated in this study are in a steady-state vibration and governed by the Helmholtz equation:

$$
\nabla^{2} p(x)+k^{2} p(x)+f(x)=0
$$

where $p(x)$ denotes the sound pressure, $f(x)$ the distributed source term, and $k$ the wave number. Denoting $C_{0}$ by the sound velocity, the wave number $k$ is expressed by using the angular velocity $\omega$ as

$$
k=\frac{\omega}{C_{0}}
$$

The boundary conditions are prescribed as

$$
\begin{gathered}
p(x)=\bar{p}(x) \\
q(x)=\frac{\partial p}{\partial n}(x)=\bar{q}(x)
\end{gathered}
$$

where $q(x)$ is related to the outward normal velocity of a particle $v(x)$ and the mass density $\rho$ as follows:

$$
q(x)=-\mathrm{i} \omega \rho v(x)
$$

\subsection{Regularized boundary integral equation}

Under the assumption of a single point sound source with intensity $I$ at the point $x^{s}$, the boundary integral equation can be expressed in a regularized form [6] as

$$
\int_{\Gamma}\left\{q^{*}(x, y)-Q^{*}(x, y)\right\} p(x) d \Gamma(x)+\int_{\Gamma} Q^{*}(x, y)\{p(x)-p(y)\} d \Gamma(x)
$$

WIT Transactions on Modelling and Simulation, Vol 44, (C) 2007 WIT Press

www.witpress.com, ISSN 1743-355X (on-line) 


$$
=-\mathrm{i} \omega \rho \int_{\Gamma} p^{*}(x, y) v(x) d \Gamma(x)+I p^{*}\left(x^{s}, y\right)
$$

The fundamental solutions which are denoted by an asterisk are given by

$$
\begin{gathered}
p^{*}(x, y)=\frac{1}{4 \pi r} \exp (-\mathrm{i} k r) \\
q^{*}(x, y)=-\frac{1}{4 \pi r^{2}}(1+\mathrm{i} k r) \exp (-\mathrm{i} k r) \frac{\partial r}{\partial n}(x) \\
Q^{*}(x, y)=-\frac{1}{4 \pi r^{2}} \frac{\partial r}{\partial n}(x)
\end{gathered}
$$

where $r$ denotes the distance between the source point $y$ and a field point $x$.

The boundary integral equation (6), which is called in this paper "OBIE", is usually applied to the standard analysis of acoustic fields and in most cases obtain successful results. Unfortunately, however, in the acoustic fields to be studied in this paper, Eq.(6) suffers the fictitious eigenfrequency problem, and gives "ghost" solutions at an infinite number of eigenfrequencies for internal subdomains without vibration. To improve this situation, we have to prepare another expression of the boundary integral equation and to use it together with Eq.(6).

\subsection{Normal derivative boundary integral equation}

We now differentiate Eq.(6) with respect to the source point $y$. Then, we can obtain the following expresssion omitting the source term:

$$
\begin{aligned}
& \int_{\Gamma}\left\{q_{, j}^{*}(x, y)-Q_{, j}^{*}(x, y)\right\} p(x) d \Gamma(x)+\int_{\Gamma} Q_{, j}^{*}(x, y)\{p(x)-p(y)\} d \Gamma(x) \\
& -\left\{\int_{\Gamma} Q^{*}(x, y) d \Gamma(x)\right\} p_{, j}(y)=-\mathrm{i} \omega \rho \int_{\Gamma} p_{, j}^{*}(x, y) v(x) d \Gamma(x)
\end{aligned}
$$

We take into account a uniform gradient of the sound pressure $p$ in the above expression, and regularize the boundary integral expression. Then, we can finally derive the following regularied boundary integral eqaution $[7,8]$ :

$$
\begin{aligned}
& \int_{\Gamma}\left\{\tilde{q}^{*}(x, y)-\tilde{Q}^{*}(x, y)\right\} p(x) d \Gamma(x) \\
& +\int_{\Gamma} \tilde{Q}^{*}(x, y)\left\{p(x)-p(y)-r_{m}(x, y) p_{, m}(y)\right\} d \Gamma(x) \\
& =-\mathrm{i} \omega \rho \int_{\Gamma}\left\{\tilde{p}^{*}(x, y)-\tilde{u}^{*}(x, y)\right\} v(x) d \Gamma(x) \\
& -\mathrm{i} \omega \rho \int_{\Gamma} \tilde{u}^{*}(x, y)\left\{v(x)-n_{m}(x) p_{, m}(y)\right\} d \Gamma(x)+I \tilde{p}^{*}\left(x^{s}, y\right)
\end{aligned}
$$

where $\tilde{(})=\partial() / \partial n(y)$, and the asterisked functions are related to the fundamental solution. This normal derivative boundary integral equation is called "NDBIE" in this paper. 
Together with the two boundary integral equations, we can explain the boundary element methods for solving the acoustic problems under consieration as in the following manner:

1 The standard BEM uses only OBIE for all nodal points,

2 The Burton-Miller Approach adopts OBIE $+\alpha$ NDBIE for all nodal points,

3 The present proposal employs OBIE $+\alpha$ NDBIE at a smaller number of element nodes, and at the other element nodes uses only the expression $\alpha$ NDBIE.

It is intersting to note that according to Ref. [5], we choose the coupling parameter $\alpha$ as $\alpha=\mathrm{i} / k$.

\section{Numerical results and discussion}

We shall take a breathing sphere with a uniform velocity $v$ on its whole surface, which is located in an infinite acoustic 3-D space. It is well known that the acoustic problem of the external domain with infinity involves the fictitious eigenfrequency issue. The solution by means of the usual boundary integral equation (OBIE) method is disturbed near the eigenfrequencies of the inside sphere itself.

The present study employs quadrilateral boundary elements with second-order polynominal interpolation functions as shown in Fig. 1. When the source point is located at a corner point, the element is divided into two subelements as Type A shown in Fig. 2. On the other hand, when the source point is located at a middle node, the element is divided into three sub-elements as shown as Type B in the figure. The singular integrals are evaluated by such a sub-element method [9].

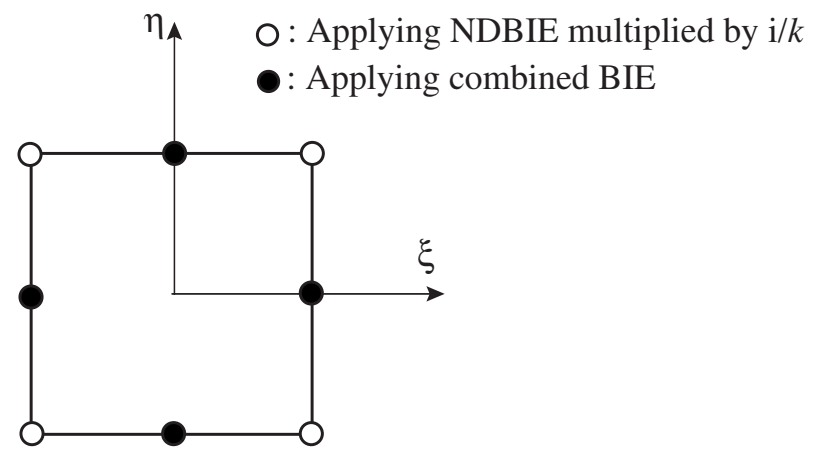

Figure 1: Quadrilateral boundary element with quadratic interpolations.

Boundary element division of the $1 / 8$ part of the spherical surface is shown in Fig. 3. Three evaluation points for the sound pressure $p$ are placed as the measuring point shown in the figure. It is assumed that the mass density $\rho=1.2\left[\mathrm{~kg} / \mathrm{m}^{3}\right]$ and the sound velocity $C_{0}=340[\mathrm{~m} / \mathrm{s}]$. 


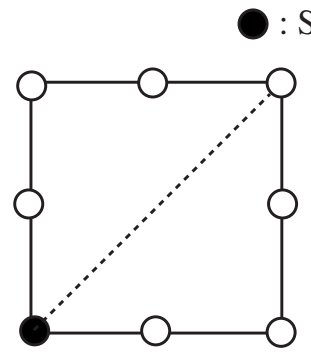

Type A

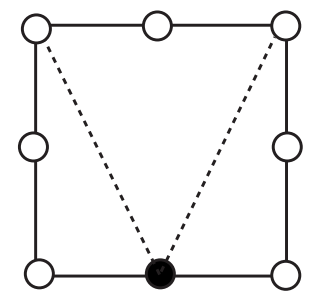

Type B

Figure 2: Types of dividing for sub-elements.
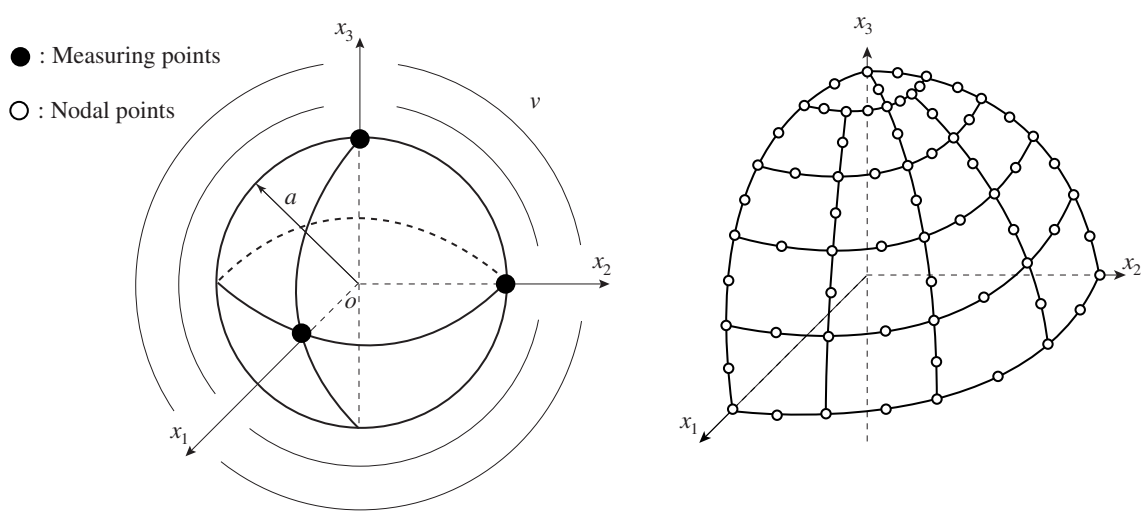

Figure 3: Analysis model 1 and boundary element discretization.

The analytical solution is available in the literature [10] and given by

$$
p(r)=v \frac{\mathrm{i} \omega \rho a^{2}}{(1+\mathrm{i} k a) r} \exp \{-\mathrm{i} k(r-a)\}
$$

Figure 4 shows the numerical results obtained by the above three methods. There seem to be the fictitious eigenfrequencies near the values of nondimensional wave numbers 3 and 6 , as the numerical solutions by the OBIE are disturbed, while the other two methods gives smooth, accurate results. Detailed views near $k a=3$, and $k a=6$ are shown in Figs. 5 and 6, respetively.

It can be seen that the present method provides accurate numerical solutions without disturbances due to the fictitious eigenfrequency issue, under a smaller amount of computational burden in comparison with the Burton-Miller method [4]. It is proved from a mathematical point of view that the present method can always provide accurate results without any disturbance due to the fictitious eigenvalue issue. 


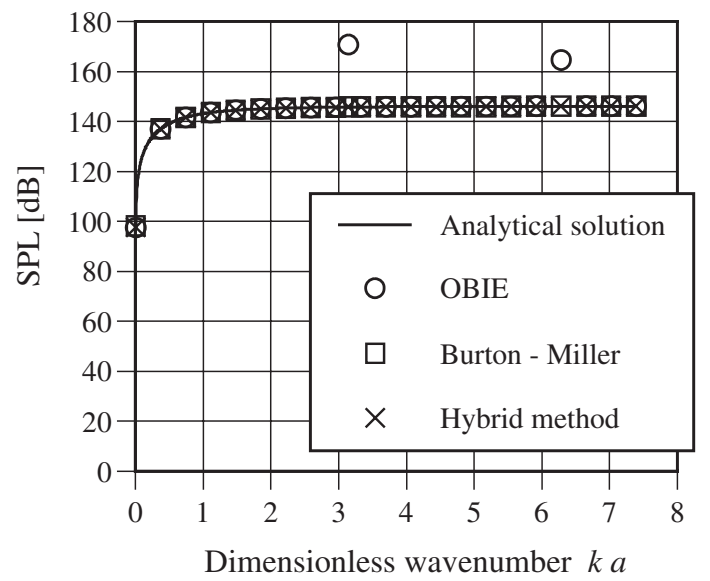

Figure 4: Numerical results obtained by OBIE, Burton-Miller and present Hybrid methods.

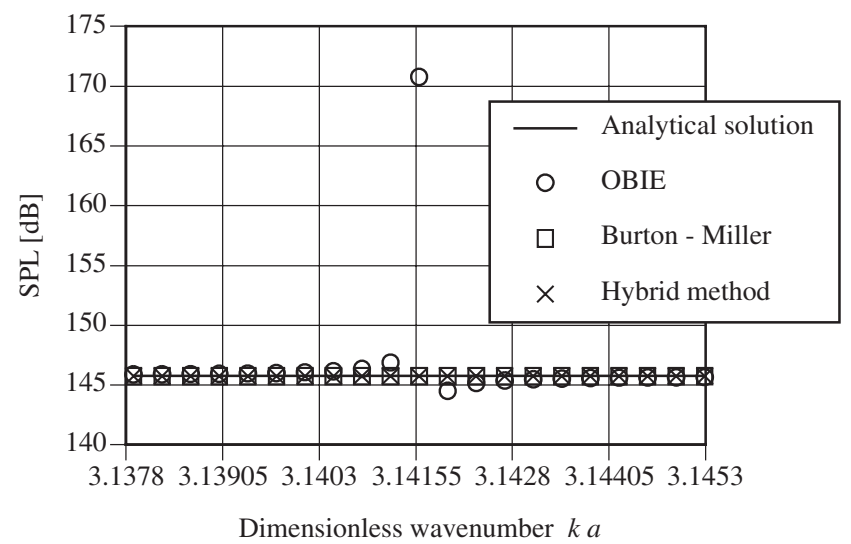

Figure 5: Detailed view of numerical results between $k a=3.1378$ and 3.1453.

Next, we shall consider the acoustic field between a rigid sphere with radius $a_{1}$ and a breathing sphere with radius $a_{2}$ concentrically located as shown in Fig. 7. In the numerical computation, it is assumed that $a_{1}=0.25 \mathrm{~m}$ and $a_{2}=0.1 \mathrm{~m}$. The boundary conditions are assumed such that the breathing sphere is subject to the same uniform normal particle velocity as the previous example and the condition on the outside spherical surface is rigid so that the particle velocity vanishes there. The 1/8 part of the two spherical boundary surfaces is divided into the same number of elements as shown in the figure. The numerical results obtained are shown in Fig. 8. Numerical computation is performed by an interval $1 \mathrm{~Hz}$ from $1 \mathrm{~Hz}$ to $2 \mathrm{kHz}$. Even in this acoustic problem, the fictitious eigenfrequency problem 


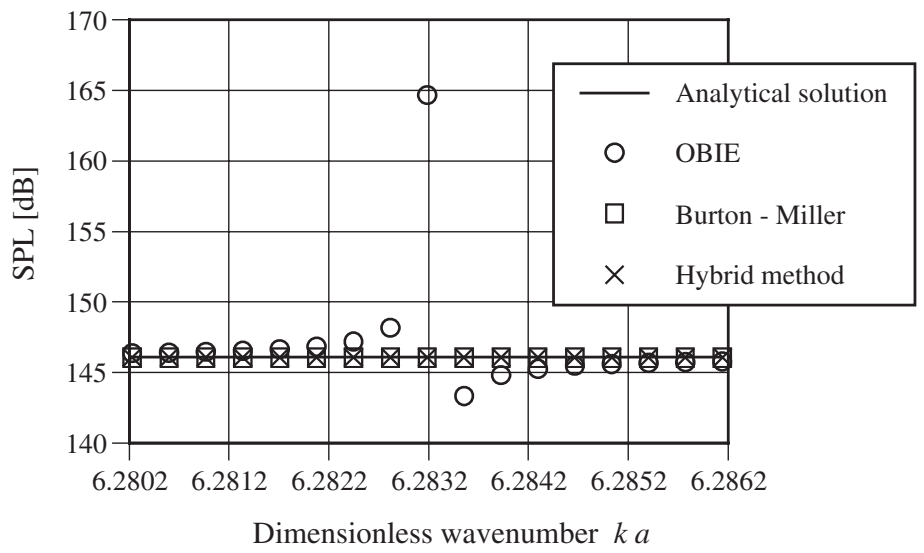

Figure 6: Detailed view of numerical results between $k a=6.2802$ and 6.2862.

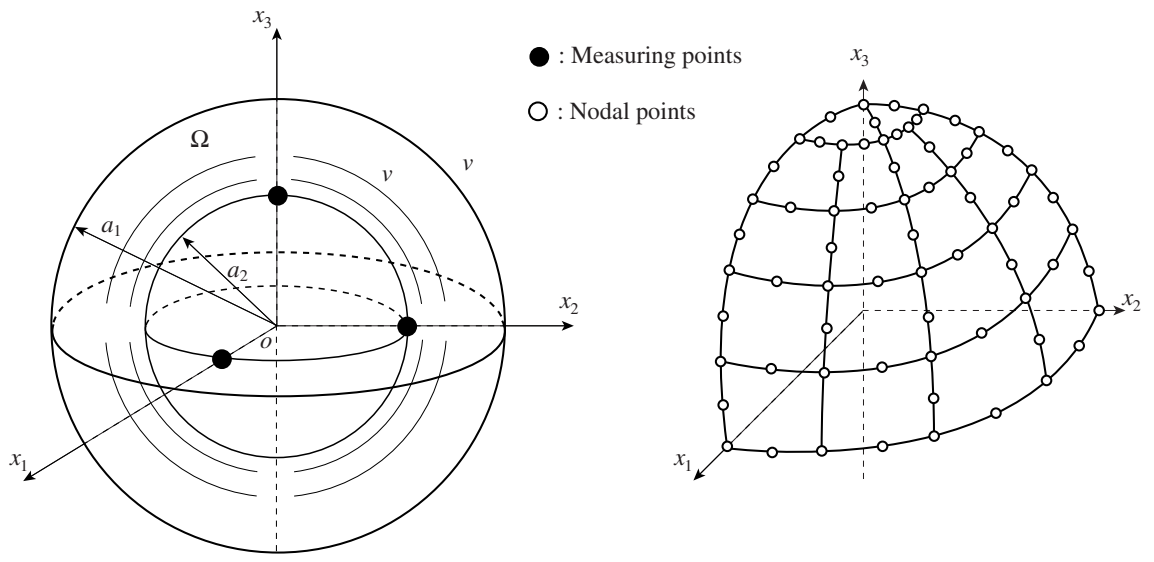

Figure 7: Analysis model 2 and its boundary element discretization.

occurs. That is, near the non-dimensional wavenumber $k a=3$ in this calculation the OBIE gives disturbed numerical results different to those by the other two methods.

Other 3-D numerical examples and analysis of 2-D acoustic fields with a few examples can be found in authors' separate papers [11,12].

\section{Concluding remarks}

The present paper has proposed an alternative approach to the Burton-Miller method to give accurate results without disturbances due to the eigenfrequency issue in the acoustic problems. The usefulness of the proposed method was demonstrated through numerical computations and comparison with other ones. 


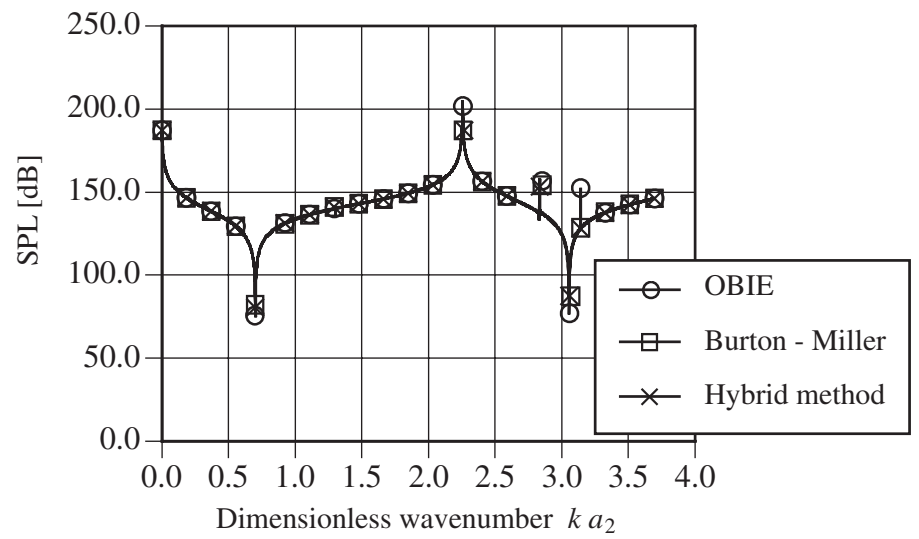

Figure 8: Numerical results obtained by OBIE, Burton-Miller and present Hybrid methods.

It is noted again that the proposed method can give the almost same results as the Burton-Miller one under a smaller burden in calculating the coefficient matrixes in the final system.

As future work along this line, it can be recommended to develop a rigorous procedure for finding the optimal shapes of acoustic fields. For such problems, it is inevitably necessary to have an analysing procedure with a reduced computational task which prevents the fictitious eigenfrequency problem from taking place.

\section{References}

[1] Tanaka, M., Matsumoto, T. \& Nakamura, M., Boundary Element Methods, Baifukan, Tokyo/Japan, 1991.

[2] Kobayashi C.S.(ed), Wave Analysis and Boundary Element Method, Kyoto University Press Online, Kyoto/Japan, 2000.

[3] Schenck, H.A., Improved integral formulation for acoustic radiation problems, J. the Acoustical Society of America, Vol.44, No.1, pp.41-58, 1968.

[4] Burton, A.J. \& Miller, G.F., The application of integral equation methods to the numerical solution of some exterior boundary-value problems, $J$. the Royal Society of London, Ser. A, Vol.323, pp.201-210, 1971.

[5] Cunefare, K.A. \& Koopmann, G., A boundary element method for acoustic radiation valid for all wavenumbers, J. the Acoustical Society of America, Vol.85, No.1, pp.39-48, 1989.

[6] Matsumoto C.T.D. \& Tanaka C.M.C. Alternative discretization technique for regularized boundary integral equation, Transactions JASCOME, Vol.1, pp.7-12, 1991. 
[7] Arai, M., Adachi, T., \& Matsumoto, H. C. Highly accurate analysis by boundary element method based on uniform gradient condition (Application for formulation of classical potential problems), Transactions of Japan Society of Mechanical Engineers (JSME), Ser. A, Vol.61, No.581, pp.161168, 1995.

[8] Matsumoto C.T.D. \& Tanaka, M. C. Evaluation of the hypersingular and regularized boundary integral equations for the boundary potential gradients in 2D fieldC Transactions JSME, Ser. A, Vol.64, No.619, pp.743-750, 1998.

[9] Yuuki C.R.D. \& Kisu C.H., Boundary Element Methods for Elastic Analysis, Baifukan, Tokyo/Japan, 1987.

[10] Itou, T., Basic Acoustical Engineering, Vol.1, Corona, Tokyo/Japan, pp.268270, 1990.

[11] Arai, Y., Tanaka, M. \& Matsumoto, T., New boundary element analysis of 3$\mathrm{D}$ acoustic fields avoiding the fictitious eigenfreqency problem, Transactions JSME, Ser. $C$, to be printed.

[12] Tanaka C.M., Matsumoto C.T. \& Arai, Y., A boundary element analysis for avoiding the fictitious eigenfrequency problem in acoustic field (2nd report: Revised version)C Transactions JSME, Ser. C, Vol.72, No.719, pp.20082093, 2006. 